\title{
1 CLE42 Delays Leaf Senescence by Antagonizing Ethylene Pathway in Arabidopsis
}

2 Yi Zhang ${ }^{1,2, *}$, Shuya Tan ${ }^{1, *}$, Yuhan $\mathrm{Gao}^{3}$, Chengcheng Kan ${ }^{1}$, Hou-Ling Wang ${ }^{1}$, Qi Yang ${ }^{1}$, Xinli Xia ,

3 Takashi Ishida ${ }^{4}$, Shinichiro Sawa ${ }^{5}$, Hongwei Guo ${ }^{2}$, and Zhonghai $\mathrm{Li}^{1}$

$4{ }^{1}$ National Engineering Laboratory for Tree Breeding, College of Biological Sciences and 5 Technology, Beijing Forestry University, Beijing, 100083, China; ${ }^{2}$ Institute of Plant and Food 6 Science, Department of Biology, School of Life Sciences, Southern University of Science and 7 Technology (SUSTech), Shenzhen 518055, Guangdong, China; ${ }^{3}$ The State Key Laboratory for 8 Biology of Plant Disease and Insect Pests, Institute of Plant Protection, Chinese Academy of 9 Agricultural Sciences, Beijing 100193, China; ${ }^{4}$ International Research Organization for Advanced 10 Science and Technology (IROAST), Kumamoto University, Kumamoto 860-8555, Japan; ${ }^{5}$ Graduate 11 School of Science and Technology, Kumamoto University, Kumamoto 860-8555, Japan

12 Author for correspondence:

13 Zhonghai Li

14 Email: lizhonghai@bjfu.edu.cn

$15 *$ These authors contributed equally to this work. 


\section{Summary}

18 Leaf senescence is the final stage of leaf development and is influenced by numerous internal and environmental factors. CLE family peptides are plant-specific peptide hormones that regulate various

20 developmental processes. However, the role of CLE in regulating leaf senescence remains unclear.

21 Here, we found that CLE42 is a negative regulator of leaf senescence by using a CRISPR/Cas9produced CLE mutant collection. The cle42 mutant displayed earlier senescence phenotypes, while overexpression of CLE42 delayed age-dependent and dark-induced leaf senescence. Moreover,

24 application of the synthesized 12-aa peptide (CLE42p) also delayed leaf senescence under natural and dark conditions. CLE42 and CLE41/44 displayed functional redundancy in leaf senescence, and the cle41 cle42 cle44 triple mutant displayed more pronounced earlier senescence phenotypes than any single mutant. Analysis of differentially expressed genes obtained by RNA-Seq methodology revealed that ethylene pathway was suppressed by overexpressing CLE42. Moreover, CLE42 suppressed ethylene biosynthesis and thus promoted the protein accumulation of EBF, which in turn decreased the function of EIN3. Accordingly, mutation of EIN3/EIL1 or overexpression of EBF1 suppressed the earlier senescence phenotypes of the cle 42 mutant. Together, our results reveal that the CLE peptide hormone regulates leaf senescence by communicating with ethylene pathway. 


\section{Introduction}

Plant leaves capture energy from sunlight to fix carbon in glucose, which is the source of food for most organisms on earth (Hohmann-Marriott \& Blankenship, 2011). As leaves age, their photosynthetic capacity gradually decreases, accompanied by the catabolism of macromolecules, including nucleic acids, proteins, and lipids. The nutrients released from senescent leaves are recycled to other developing organs, such as young leaves, flowers, and seeds, leading to an increase in reproductive success (Guo \& Gan, 2005; Lim et al., 2007). Efficient senescence is essential for maximizing viability in the next generation or season, whereas premature senescence caused by various internal or environmental signals reduces the yield or fresh product quality of agronomically important crop plants (Hortensteiner \& Feller, 2002). Thus, the appropriate onset and progression of leaf senescence are critical for plant fitness (Uauy et al., 2006).

Leaf senescence is not a passive process but a genetically controlled developmental process (Nam, 1997; Guo \& Gan, 2005; Lim \& Nam, 2005). In model plants such as Arabidopsis, tomato, and rice, a number of components in the regulation of leaf senescence were identified by forward genetic screenings (Lim et al., 2007; Woo et al., 2016; Li et al., 2020). Through multi-omics studies such as genomics, transcriptomics, proteomics, and metabolomics, thousands of senescenceassociated genes (SAGs) were identified in dozens of plant species (Woo et al., 2013; Kim et al., 2016). Reverse genetics studies, including the construction of gain- or loss-of-function mutants, have also identified hundreds of functional SAGs (Li et al., 2020).

Although leaf senescence is mainly controlled by developmental age, it is also influenced by an array of internal and external signals (Guo \& Gan, 2005; Lim et al., 2007). Plant hormones are major players regulating various stages of leaf senescence, including the onset, progression, and terminal phase of senescence. Ethylene, jasmonic acid (JA), abscisic acid (ABA), salicylic acid (SA), and strigolactone promote leaf senescence, whereas cytokinin, auxin, and gibberellic acid (GA) delay this process (Gan \& Amasino, 1995; Lim et al., 2007; Woo et al., 2019). Among them, ethylene plays an important regulatory role in leaf senescence and fruit ripening (Abeles et al., 1988). Exogenous application of ethylene promotes leaf senescence, while spraying ethylene synthesis or signaling inhibitors delays senescence (Abeles et al., 1988). Forward genetic screening revealed that EIN2 (ETHYLENE-INSENSITIVE2), the core member of the ethylene signaling pathway, is a positive regulator of leaf senescence (Oh et al., 1997). Mutation of EIN2 delayed leaf senescence (Oh et al., 1997; Kim et al., 2009), while overexpression of EIN2 promoted this process (Li et al., 2014). EIN3, a master transcription factor in the ethylene pathway, is also a positive regulator of leaf senescence (Li et al., 2013). EIN3 directly binds to the promoter of $m i R 164$ and inhibits its expression, thereby indirectly promoting the expression of $O R E 1$, a core component in the regulation of leaf senescence (Li et al., 2013; Kim et al., 2014). In addition, EIN3 directly regulates the expression of chlorophyll 
catabolic genes and promotes leaf senescence (Qiu et al., 2015). Ethylene also interacts with JA, SA, and strigolactone signaling pathways to coordinate the leaf senescence process (Li et al., 2013; Ueda \& Kusaba, 2015; Wang et al., 2021; Yu et al., 2021).

Plant polypeptide hormones are a class of small molecule signaling substances that play an important role in regulating plant growth and development and the response to environmental stresses (Fiers et al., 2007; Betsuyaku et al., 2011; Hirakawa \& Sawa, 2019). Since the discovery of the small peptide hormone systemin in tomato (Pearce et al., 1991), an increasing number of plant peptide signaling molecules have been identified (Hirakawa \& Sawa, 2019). The CLAVATA3 (CLV3)/ENDOSPERM SURROUNDING REGION (ESR) (CLE) peptide family is one of the largest families of plant peptide hormones (Fletcher, 2020). The CLE gene encodes a precursor protein with a full length of approximately 100 amino acids (aa), which is processed by an unknown protease to form a mature peptide with a length of 12 to 14 aa (Ito et al., 2006; Fletcher, 2020). CLE mature peptides bind to different membrane-bound receptors and thus transduce signals into the cell to regulate plant development by modulating downstream transcription factors or communicating with canonical plant hormones (Etchells et al., 2012; Gao \& Guo, 2012; Zhang et al., 2016; Mou et al., 2017; Fletcher, 2020). CLE family members are expressed in multiple tissues and are involved in regulating various aspects of plant development (Fiers et al., 2007; Yamaguchi et al., 2016), but their role in leaf senescence remains largely unclear.

A major problem in dissecting the functions of peptide hormones is the lack of loss-of-function mutants generated by canonical mutagenesis methods due to their small gene size (Yamaguchi et al., 2017). Fortunately, CRISPR/Cas9-mediated gene editing technology overcomes this difficulty and has been used to generate loss-of-function mutants of CLE family genes (Yamaguchi et al., 2017). Using a CRISPR/Cas9-produced CLE mutant collection, we found that the leaves of CLE42 loss-offunction mutants senesced prematurely, whereas overexpression of CLE42 significantly delayed the leaf senescence process, suggesting that CLE42 is a negative regulator of senescence. Transcriptome and genetic analyses revealed that CLE42 delays leaf senescence by suppressing the ethylene pathway. 
Materials and Methods

Plant materials and growth conditions

100

101

102

103

104

105

106

107

108

109

110

Arabidopsis thaliana Columbia-0 (Col-0) was used as the wild type in this study. The CRISPR/Cas9produced CLE mutant collection was described previously (Yamaguchi et al., 2017). The plant materials pxy-3 and pxy pxl1 pxl2 (Smit et al., 2020), ein3-1 eill-1 (Alonso et al., 2003), and ACS octuple mutant (acs2-1 acs4-1 acs5-2 acs6-1 acs7-1 acs9-1 amiRacs8 acs11) (Tsuchisaka et al., 2009) used in this study were described previously. Seeds were surface-sterilized and plated on Murashige and Skoog (MS) medium (4.3 g/L MS salts, $1 \%$ sucrose [pH 5.7 to 5.8], and $8 \mathrm{~g} / \mathrm{L}$ agar). The plates were then stored for $2 \mathrm{~d}$ at $4{ }^{\circ} \mathrm{C}$ before exposing them to white light (PAR 100 to $150 \mu \mathrm{E}$ $\mathrm{m}^{-2} \mathrm{~s}^{-2}$ ). After 3-4 d, light-grown seedlings were transferred to soil and grown at $22{ }^{\circ} \mathrm{C}$ under a $16 \mathrm{~h}$ light/8 h dark cycle (PAR 100 to $150 \mu \mathrm{E} \mathrm{m}^{-2} \mathrm{~s}^{-2}$ ). For the triple response assay, genotypes were grown on MS medium supplied with or without the indicated concentration of 1-aminocyclopropane-1carboxylic acid (ACC) (Sigma-Aldrich, CAS 22059-21-8).

\section{Plasmid construction and transformation}

To generate 35S:GFP-CLE42/Col-0, the full-length CLE42 cDNA sequence was amplified and then inserted into the pEGAD vector (Cutler et al., 2000). To construct the $\beta$-estradiol inducible CLE42 overexpression lines (iCLE42), the CLE42 (linked with $3 \times$ Flag) CDS was amplified and then introduced into the pER8 vector (Zuo et al., 2000). Amplified fragments were inserted into the respective vectors by using the In-Fusion enzyme (Takara Bio, USA). For construction of CRISPR/cas9-mediated CLE42 knockout plants, a target site of CLE42 designed using CRISPR-P (http://crispr.hzau.edu.cn/CRISPR2/) was used (Table S1), and the homozygous plants were identified by sequencing. All constructs were transformed into Agrobacterium tumefaciens cells (strain GV3101), which were then used to transform Col-0 plants using the floral dip method (Clough \& Bent, 1998). All primer sequences used here are listed in Table S1.

\section{Leaf senescence assays under natural and stress conditions}

All senescence experiments were performed using the third and fourth rosette leaves. Age-dependent leaf senescence was evaluated as described previously (Woo et al., 2001). Leaves of individual plants at the indicated developmental stages were detached, photographed, and used to assess the chlorophyll content, the photochemical efficiency of PSII (Fv/Fm) and $S A G$ expression. For the hormone-, salt-, or dark-induced leaf senescence assay, leaves at $12 \mathrm{~d}$ after emergence (DAE) were detached and floated on $5 \mathrm{mM}$ MES buffer ( $\mathrm{pH}$ 5.7) in the presence of hormone or $\mathrm{NaCl}$ at $22{ }^{\circ} \mathrm{C}$ in the dark (Woo et al., 2004).

\section{RNA extraction and quantitative Real-Time PCR analysis}

Total RNA was extracted using plant RNA kits (ER301; TransGen Biotech, China), and cDNA was synthesized using a cDNA synthesis kit (AT341; TransGen Biotech, China). Quantitative RT-PCR 
(qPCR) analysis was performed to determine gene expression levels using an ABI7500 Real-Time PCR system with a qPCR kit (W812014, Welab Biotech, China), and then relative mRNA quantities were calculated from the average values using the $\Delta \Delta \mathrm{C}_{\mathrm{T}}$ method (Schmittgen \& Livak, 2008). All primers were shown in Table $\mathrm{S} 1$.

\section{RNA-sequencing analysis}

The third and fourth rosette leaves of 24-d-old Col-0 and CLE42ox plants were collected and ground into a powder in liquid nitrogen. All samples were collected in three biological replicates. Total RNA was extracted using a RNeasy Plant Kit (Qiagen, cat. nos. 74904), and the quality and quantity of RNA were checked using an Agilent 2100 Bioanalyzer (Agilent Technologies, Inc., Santa Clara, CA, USA). RNA-Seq data were generated with an Illumina HiSeq ${ }^{\mathrm{TM}} 2500$ sequencing platform (Biomarker Ltd., Beijing, China). Raw reads (fastq format) were trimmed and filtered using in-house Perl scripts (Biomarker Ltd, China). The reads were then mapped to the Arabidopsis reference genome using the Hisat2 algorithm. DEGs were filtered using the following criteria: |Log2 (fold change) $\mid>1.0, \mathrm{P}<0.05$. Gene Ontology (GO) enrichment analysis was performed by using the GO database (http://geneontology.org/). Default parameters were used for all bioinformatics software.

\section{Histochemical analysis of GUS activity}

GUS staining was performed as previously described (Jefferson et al., 1987). We incubated tissues or seedlings with GUS staining solution $\left(100 \mathrm{mM} \mathrm{Na} 3 \mathrm{PO}_{4}\right.$ [pH 7.0], $1 \mathrm{mM}$ potassium ferricyanide, 1 mM EDTA, 1\% Triton X-100, $1 \mathrm{mM}$ potassium ferrocyanide, and $1 \mathrm{mg} / \mathrm{mL}$ 5-bromo-4-chloro-3indolyl- $\beta$-D-glucuronide) overnight at $37^{\circ} \mathrm{C}$ and then incubated them for hours in ethanol to eliminate chlorophyll before photographing. GUS quantification was carried out using 4-methylumbelliferyl$\beta$-D-glucuronide (4-MUG; Sigma-Aldrich) as the substrate.

\section{Western blotting}

One hundred milligrams of tissues or seedlings was ground into a powder in liquid nitrogen, and 100 $\mu \mathrm{L}$ of $2 \times$ protein loading buffer was then added and boiled for $5 \mathrm{~min}$. Subsequently, $10 \mu \mathrm{L}$ per sample was analyzed by western blotting using monoclonal anti-GFP antibodies conjugated to horseradish peroxidase (HRP; Sigma-Aldrich) and separated by 8\% SDS-PAGE. Coomassie brilliant blue (CBB) was the total protein loading control.

\section{Measurement of ethylene production}

The third/fourth rosette leaves of Arabidopsis were used for quantification of ethylene production. The detached leaves treatment with or without CLE42p solution were weighed and incubated in a vial at $22^{\circ} \mathrm{C}$ under a $16 \mathrm{~h} \mathrm{light} / 8 \mathrm{~h}$ dark cycle. Ethylene production after $24 \mathrm{~h}$ of incubation was measured with gas chromatography as previously described (Tsuchisaka et al., 2009; Qiu et al., 2015).

\section{Chromatin immunoprecipitation (ChIP)-qPCR}


167 Two grams of 5-d-old 35S:EIN3-GFP seedlings treated with CLE44p or ACC were used for ChIP 168 experiments as previously described (Saleh et al., 2008). GFP antibody was used to 169 immunoprecipitate the protein-DNA complex. The enrichment of DNA fragments was determined by 170 qPCR. All primers used here are listed in Table S1. 


\section{Results}

\section{CLE42 negatively regulates age-dependent and dark-induced leaf senescence}

To investigate the function of CLE family peptides in leaf senescence, we first performed a senescence phenotypic screening by using a CRISPR/Cas9-produced CLE mutant collection (Yamaguchi et al., 2017). The mutants were grown in soil under long-day conditions (16 h light/8 h dark) alongside wild-type Col- 0 . We found that the cle42 mutant displayed a premature senescence phenotype (Fig. 1a), suggesting that CLE42 is a negative regulator of leaf senescence. The CLE42 peptide and TRACHEARY ELEMENT DIFFERENTIATION INHIBITION FACTOR (TDIF; CLE41/CLE44 peptide) act as inhibitors of tracheary element differentiation (Yaginuma et al., 2011; Etchells et al., 2016). To further determine its function, we generated transgenic plants overexpressing CLE42 fused with GFP driven by the $35 S$ promoter (35S:GFP-CLE42, CLE42ox), and two transgenic lines (CLE42ox \#1 and \#2) were chosen for further analysis (Fig. S1). Remarkably, the leaves of CLE42ox plants displayed delayed senescence compared to those of Col-0 and cle42 (Fig. 1a,b). We next examined in detail the senescence phenotypes of the fourth leaves of Col-0, cle42, and CLE42ox during their lifespans. The leaves of cle 42 mutants started to turn yellow from the tip at Day 22 (i.e., $22 \mathrm{~d}$ after emergence of the fourth leaves), whereas Col-0 and CLE42ox leaves turned yellow at Days 26 and 34, respectively (Fig. 1c), with lower chlorophyll contents (Fig. 1d) and photochemical efficiency of PSII (Fv/Fm) (Fig. 1e). Transcript levels of SAG12, a marker gene of leaf senescence, in the cle42 mutant were more dramatically induced than those in Col-0 and CLE42ox (Fig. 1f). Additionally, the transcripts of CLE42 and CLE41/44 decreased with leaf age (Fig. S2), raising the possibility that they act as suppressors of leaf senescence.

To further assess the role of CLE42 in leaf senescence, we examined the senescence phenotypes of cle42 and CLE42ox under dark conditions. The third rosette leaves detached from 21-d-old Col-0, cle42, and CLE42ox plants were placed in the dark. We found that cle42 exhibited significantly faster dark-induced senescence, while CLE42ox showed significantly delayed dark-induced senescence than Col-0 (Fig. 1g,h). We also tested the effects of other senescence-regulating signals, such as plant hormones, salt stress, and $\mathrm{H}_{2} \mathrm{O}_{2}$, on the senescence phenotypes of cle42 and CLE42ox. Compared with the Col-0 and cle42 mutants, the leaves of CLE42ox displayed delayed senescence phenotypes upon treatment with $\mathrm{ABA}$, JA, $\mathrm{ACC}$ (ethylene precursor), $\mathrm{NaCl}$, or $\mathrm{H}_{2} \mathrm{O}_{2}$ under dark conditions (Fig. S3). Together, these results indicate that CLE42 delays both age-dependent and dark- and stressinduced leaf senescence. 
(a)
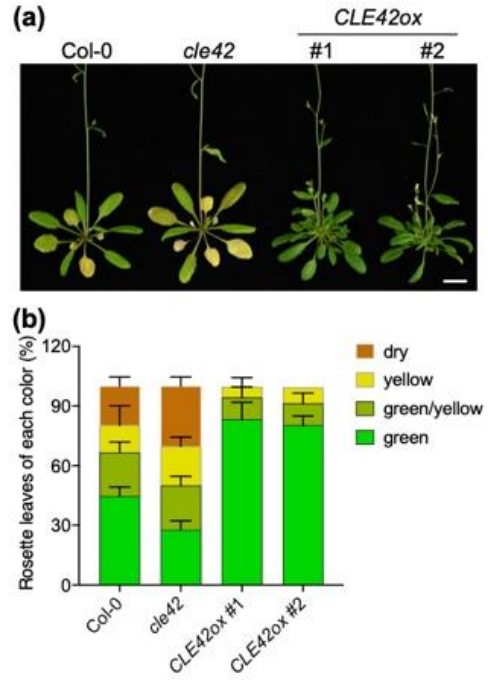

(c)

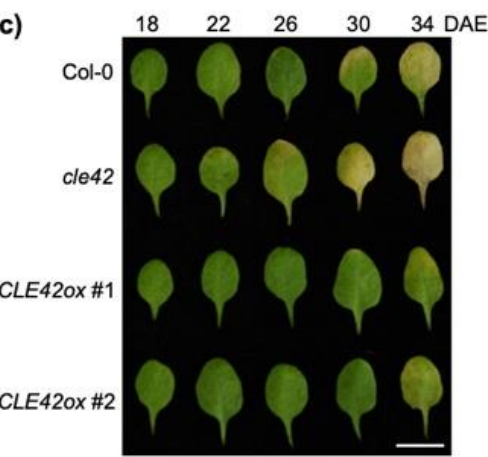

(d)

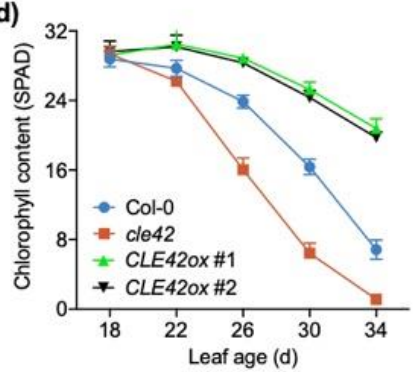

(e)

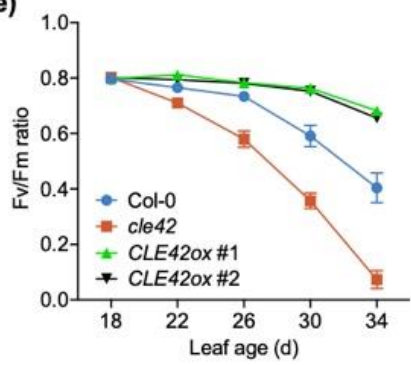

(f)

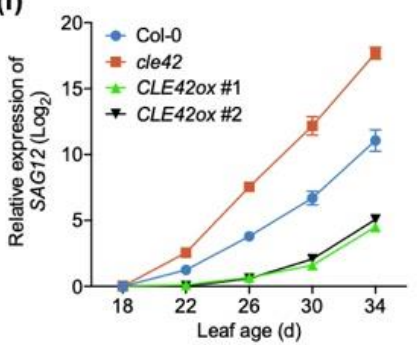

(g) $\mathrm{Col}-0 \quad$ cle $42 \quad$ CLE420x

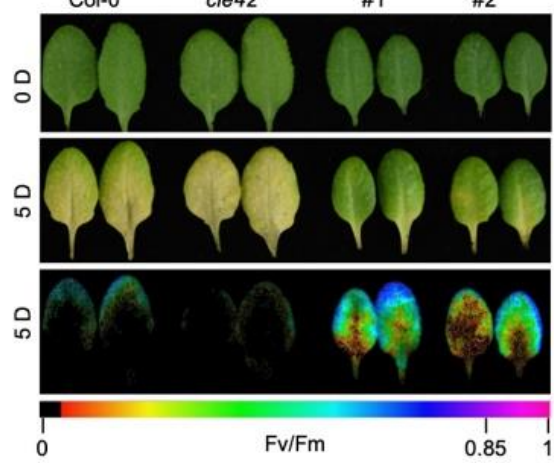

(h)

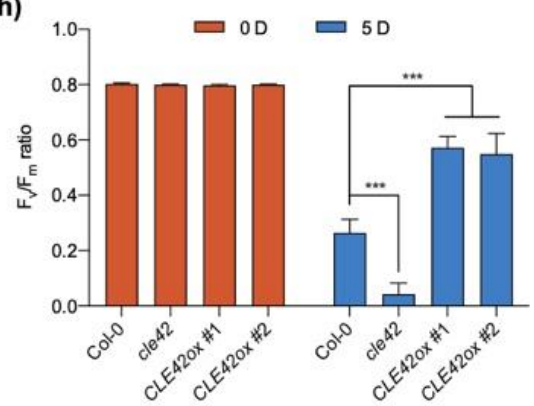

Fig. 1 CLE42 negatively regulates age-dependent and dark-induced leaf senescence. (a) The senescence phenotypes of 40-d-old wild-type Col-0, cle42, and CLE42ox. Two independent lines, CLE42ox \#1 and \#2, were examined. Bar, $1 \mathrm{~cm}$. (b) quantification of the leaf senescence phenotypes of the third/fourth rosette leaves shown in (a). (c) Phenotypes of the third/fourth rosette leaves of Col0, cle42, and CLE42ox at the indicated age. Representative leaves are shown. Bar, $1 \mathrm{~cm}$. (d-f) Chlorophyll content (d), Fv/Fm ratio (e), and SAG12 expression (f) in the leaves shown in (c). The bars indicate the mean $\pm \mathrm{SD}(\mathrm{n}=20)$. (g) The senescence phenotypes and Fv/Fm ratio of Col-0, cle42, and CLE42ox leaves upon dark treatment for 4 days. (h) Fv/Fm ratio of leaves shown in (g). The bars indicate the mean $\pm \mathrm{SD}(\mathrm{n}=20) .{ }^{*} * * \mathrm{P}<0.001$, two-way ANOVA.

\section{CLE41/44 and PXY are involved in leaf senescence}

215 The mature form of CLE42 is a dodecapeptide (HGVPSGPNPISN) and differs from CLE41/CLE44 216 (HEVPSGPNPISN) only in the second position. Both forms play an essential role in regulating cell 217 division in vascular meristems by binding to the leucine-rich repeat receptor kinase (LRR-RK) PXY 218 (PHLOEM INTERCALATED WITH XYLEM) (Fisher \& Turner, 2007; Hirakawa et al., 2008; 219 Zhang et al., 2016). To examine whether there is genetic redundancy between CLE42 and CLE41/CLE44 in regulating leaf senescence, we generated cle41 cle42 cle44 triple mutants by crossing (Fig. S4). The onset of leaf senescence started earlier in cle 41 cle 42 cle 44 triple mutants than 
in any single mutant (Fig. 2a), with a pronounced decrease in the chlorophyll content and Fv/Fm as well as an earlier induction of $S A G 12$ expression (Fig. 2b-d), indicative of the existence of genetic redundancy. Interestingly, the cle 42 mutant displayed stronger senescence phenotypes than cle 41 and cle44, with the lowest chlorophyll contents and Fv/Fm (Fig. 2b,c), suggesting that CLE42 plays a dominant role in leaf senescence. As expected, mutation of $P X Y$ accelerated leaf senescence (Fig. 2a), suggesting that the CLE41/42/44-PXY module prevents leaf from premature senescence in Arabidopsis.

In Arabidopsis, two kinases, PXY-like 1 (PXL1) and PXL2, are closely related to PXY (Fisher \& Turner, 2007), and the CLE41/42/44 peptides also interact with PXL1/2 (Zhang et al., 2016; Mou et al., 2017). Single mutants of pxll and pxl2 did not exhibit obvious phenotypic changes in the vascular stem; however, the triple mutant pxy pxll pxl2 showed enhanced alterations of the vascular phenotype compared with that of pxy (Etchells et al., 2013), indicating that PXL1 and PXL2 function redundantly with PXY in the regulation of vascular tissue development. Unexpectedly, no evidently accelerated senescence phenotype was observed in pxy pxl1 pxl2 leaves in comparison with pxy leaves (Fig. S5), indicating that PXL1 and PXL2 play a minor role in regulating leaf senescence. Interestingly, the leaves of cle 41 cle 42 cle 44 showed stronger earlier senescence symptoms than those of pxy or pxy pxl1 pxl2, with lower chlorophyll contents and $\mathrm{Fv} / \mathrm{Fm}$, as well as earlier induction of SAG12 expression (Fig. 2b-d; Fig. S5), suggesting that CLE41/CLE42/CLE44 regulates leaf senescence in a PXY-dependent and PXL-independent manner.

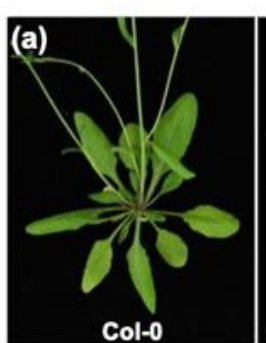

(b)

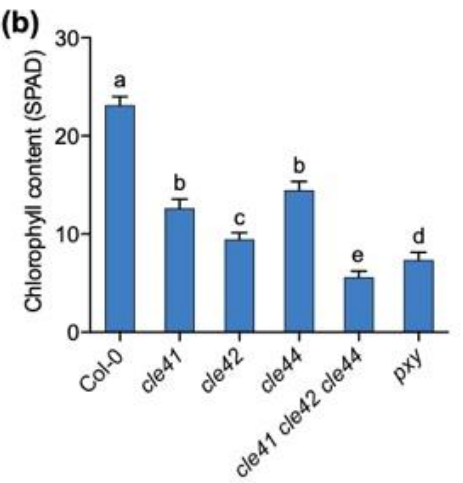

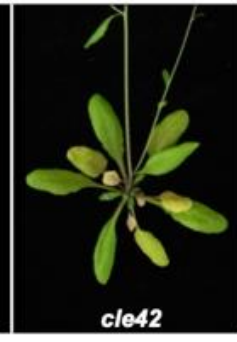

(c)

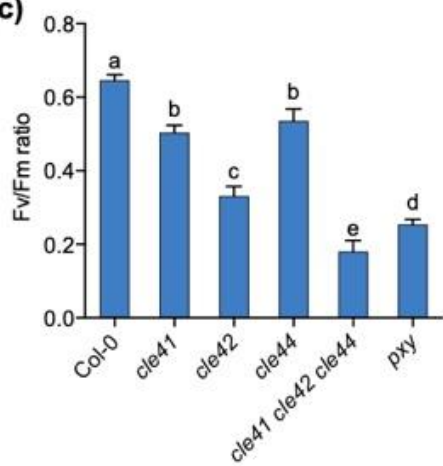

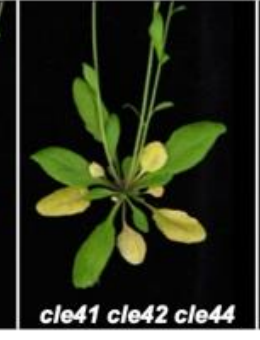

(d)

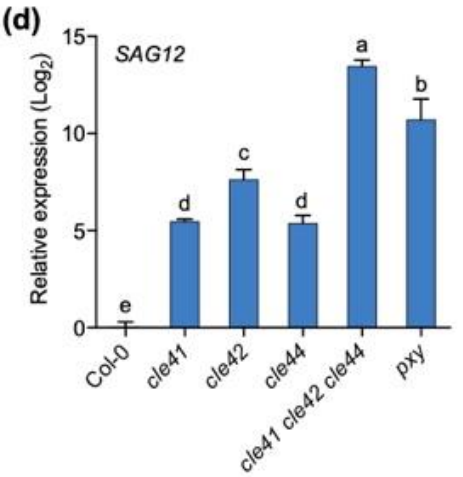

Fig. 2 CLE41/42/44-PXY module is involved in leaf senescence. (a) The senescence phenotypes of 35-d-old Col-0, cle41, cle42, cle44, cle41 cle42 cle44, and pxy. Bar, $1 \mathrm{~cm}$. (b-d) Chlorophyll content (b), Fv/Fm ratio (c), and SAG12 expression (d) in the fourth leaves shown in (a). Different letters above the bars indicate statistically significant differences (adjusted $\mathrm{P}<0.05$, one-way ANOVA). 


\section{CLE42p delays age-dependent and dark-induced leaf senescence}

247 To determine whether the mature form of the CLE42 peptide is enough to function as a regulator of 248 leaf senescence, we synthesized a 12-aa peptide (CLE42p, HGVPSGPNPISN) derived from the CLE domain of the CLEL42 precursor. Wild-type Col-0 and cle42 plants were sprayed with water (Mock) or $5 \mu \mathrm{M}$ CLE42p every 4 days. Remarkably, exogenous application of CLE42p significantly delayed leaf senescence in Col-0 plants and compensated for earlier senescence of cle42 mutants (Fig. 3a).

252 Time-course analyses revealed that treatment with CLE42p evidently delayed the leaf senescence 253 process in Col-0 and cle42 backgrounds, with higher levels of chlorophyll contents and $\mathrm{Fv} / \mathrm{Fm}$ 254 compared with the controls (Fig. 3b,c). Interestingly, the cle42 mutant displayed senescence 255 phenotypes similar to those of Col-0 upon treatment with CLE42p (Fig. 3a-c), implying that in vitro synthesized CLE42p is biologically functional and rescues the phenotypes of cle 42 . In contrast, the pxy mutant exhibited earlier leaf senescence phenotypes, with lower chlorophyll content and Fv/Fm, which was slightly rescued by CLE42p, implying that CLE42 regulates leaf senescence mainly via the receptor PXY. Inducible overexpression of CLE42 delayed leaf senescence in Col-0 but not in the pxy background, thus supporting our hypothesis (Fig. 3d-f).

We also examined the effect of CLE42p on leaf senescence under dark conditions. Compared with the controls, CLE42p evidently delayed the dark-induced senescence process in Col-0 and cle 42 backgrounds (Fig. 3g), with high levels of chlorophyll content and Fv/Fm (Fig. 3h,i), while slight influence was observed in $p x y$ leaves. Similarly, synthetic CLE41/44p (HEVPSGPNPISN) was also able to restore the phenotypes of the cle42 mutant under dark conditions (Fig. S6), which further suggests that there is functional redundancy between CLE42 and CLE41/44. Together, our data demonstrated that exogenous application of synthetic CLE42p is sufficient to delay both agedependent and dark-induced leaf senescence. 


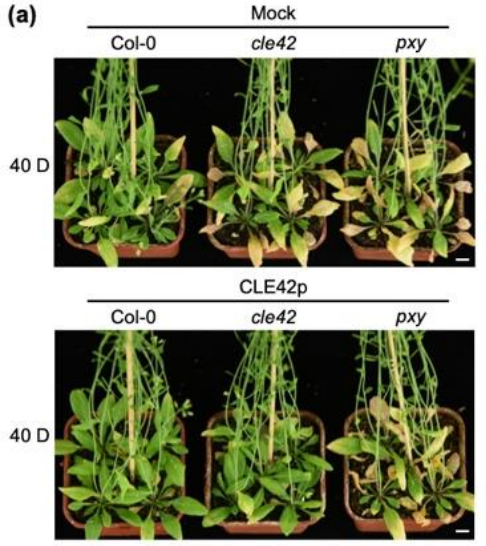

(d)

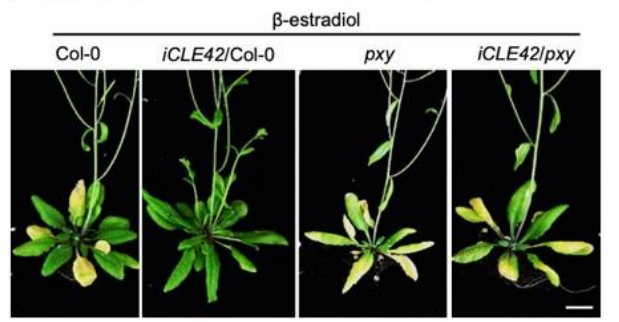

(g)

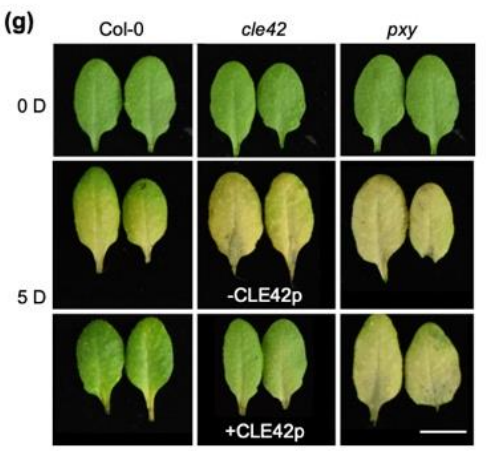

(b)
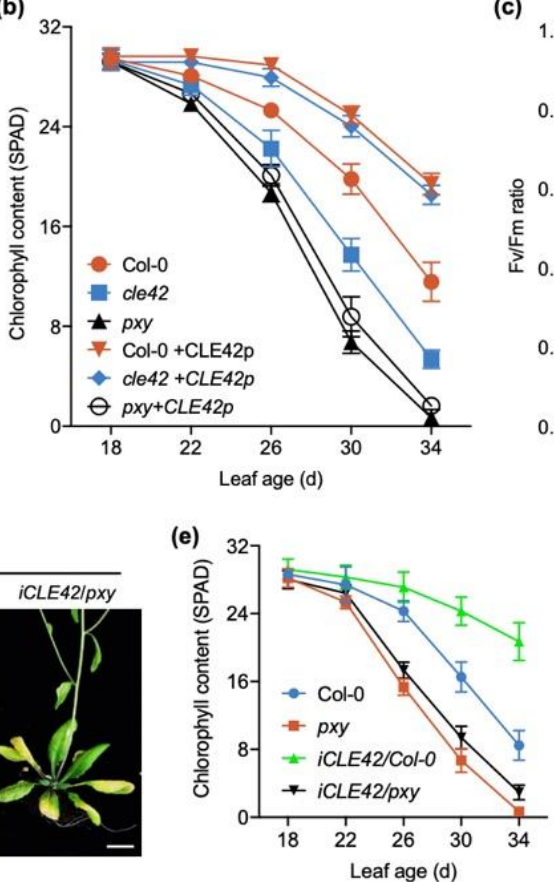

(c)

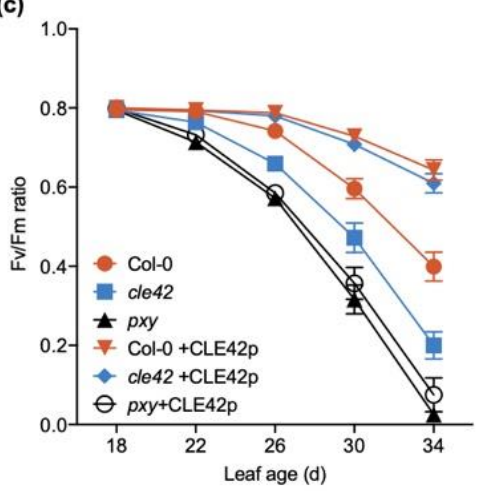

(f)

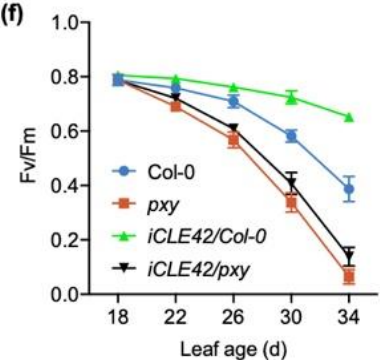

269
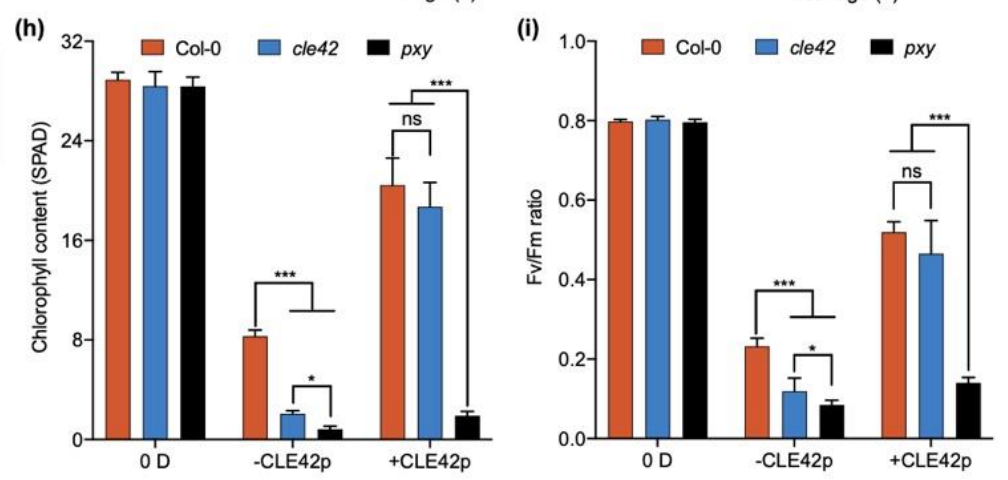

Fig. 3 Exogenous application of CLE42p delays age-dependent and dark-induced leaf senescence. (a) The senescence phenotypes of 40-d-old wild-type Col-0 and cle42 upon treatment with CLE42p. Col-0 and cle 42 were germinated on MS containing water (Mock) or $10 \mu \mathrm{M}$ CLE42p, and the 5-dayold seedlings were transferred to soil and sprayed with CLE42p every $4 \mathrm{~d}$. Bars, $1 \mathrm{~cm}$. (b) and (c) $\mathrm{Fv} / \mathrm{Fm}$ ratio (b) and chlorophyll content (c) in the leaves shown in (a) at the indicated age. The bars indicate the mean $\pm \mathrm{SD}(\mathrm{n}=20)$. (d) The senescence phenotypes of 40-d-old Col-0, iCLE42/Col-0, pxy, and iCLE42/pxy plants upon treatment with $\beta$-estradiol. Seeds were germinated on MS medium containing $10 \mu \mathrm{M} \beta$-estradiol, and 5-d-old seedlings were transferred to soil and then sprayed with 10 $\mu \mathrm{M} \beta$-estradiol every 4 days. Bar, $1 \mathrm{~cm}$. (e) and (f) Chlorophyll content (e) and Fv/Fm ratio (f) in the leaves of Col-0, iCLE42/Col-0, pxy, and iCLE42/pxy plants at the indicated age. (g) The senescence phenotypes and $\mathrm{Fv} / \mathrm{Fm}$ ratio of Col-0 and cle42 leaves upon treatment with CLE42p under darkness for 4 days. Bars, $1 \mathrm{~cm}$. (h) and (i) chlorophyll content (h) and $\mathrm{Fv} / \mathrm{Fm}$ ratio (i) in the leaves shown in (g). The bars indicate the mean $\pm \mathrm{SD}(\mathrm{n}=20) . * \mathrm{P}<0.05, * * * \mathrm{P}<0.001$, two-way ANOVA.

\section{Transcriptome analysis reveals the CLE42-mediated signaling pathway}


To reveal the molecular basis of CLE42-regulated leaf senescence, we performed transcriptome analysis of 21-day-old leaves of Col-0 and CLE42ox plants. Compared with Col-0, 2,495 differentially expressed genes (DEGs) were identified in CLE42ox leaves $\left(\left|\log _{2}(\mathrm{FC})\right|>1, \mathrm{FDR}<0.05\right)$ (Dataset S1). Gene Ontology (GO) enrichment analysis of the DEGs showed that the upregulated genes were highly enriched in the regulation of meristem growth and cell size, cell wall biogenesis, auxin polar transport, and xylem development (Fig. S7a), which is consistent with previous findings that TDIFs are involved in regulating meristem and xylem development. The downregulated genes were related to defense responses and hormone signaling pathways, such as JA, SA, and ethylene (Fig. S7b), suggesting that CLE42 delays leaf senescence by suppressing hormone signaling. In agreement with the delayed senescence phenotype of CLE42ox, many SAGs were downregulated, such as SAG12, SAG13, ORE1, WRKY75, WRKY53, and YLS9 (Fig. S8).

A previous study showed that the TDIF-PXY signaling pathway may interact and suppress ET signaling in vascular development, as many ET-responsive factors were upregulated in the pxy mutant by microarray expression analysis (Etchells et al., 2012). The triple response phenotype is commonly used as an ethylene-specific growth response in Arabidopsis and refers to the exaggerated apical hooks, shortened hypocotyls, and roots of dark-grown seedlings exposed to ethylene or treated with the ethylene precursor ACC (Bleecker et al., 1988). Overexpression of CLE42 conferred significant attenuation of triple response phenotypes, with longer hypocotyls, compared with wild-type seedlings upon treatment with low concentrations of ACC (0.1-5 $\mu \mathrm{M})$ (Fig. S9). In contrast, seedlings of cle42 mutants displayed an enhanced response to low concentrations of ACC (0.1-5 $\mu \mathrm{M})$ (Fig. S9), suggesting that CLE42 is involved in the ethylene signaling pathway. Notably, although seedlings of cle 41, cle 42 and cle44 single mutants showed enhanced sensitivity to ethylene compared with Col-0, the triple response of cle42 seedlings was the most obvious (Fig. S10), implying that CLE42 may act as a prominent player in the communication between TDIF-PXY and the ethylene signaling pathway.

\section{CLE42 decreases the function of EIN3 by promoting the protein accumulation of EBF}

311 Because EIN3 is a master transcription factor of the ethylene signaling pathway and a positive regulator of leaf senescence, we next investigated the effect of CLE42p treatment on its function. To this end, two transgenic lines that harbor the GUS reporter gene driven by five tandem repeats of the EIN3 binding site (EBS) followed by the minimal $35 \mathrm{~S}$ promoter, $5 \times E B S: G U S / \mathrm{Col}-0$ and $5 \times E B S: G U S / E I N 3 o x$ (Stepanova et al., 2007), were used to examine the function of EIN3 in the absence or presence of CLE42p. As expected, a high level of EIN3 function was observed in the seedlings of $5 \times E B S: G U S / E I N 3 o x$ in comparison with $5 \times E B S: G U S /$ Col-0 (Fig. 4a,b). Interestingly, the functions of EIN3 were significantly reduced in the seedlings of $5 \times E B S: G U S / C o l-0$ and $5 \times E B S: G U S / E I N 3$ ox upon treatment with CLE42p compared with the controls (Fig. 4a), which was 
further supported by the quantification assays of GUS activity (Fig. 4b). These data suggest that CLE42 suppresses the function of EIN3.

We next investigated how EIN3 function is modulated by CLE42p. To this end, we monitored the level of EIN3 protein using 35S:EIN3-GFP/Col-0 plants with anti-GFP antibody in response to CLE42p treatment. In agreement with previous findings, the level of EIN3 protein dramatically increased upon treatment with 1-aminocyclopropane-1-carboxylic acid (ACC), a key precursor of ethylene biosynthesis. In contrast, the protein accumulation of EIN3 was evidently decreased in the presence of CLE42p (Fig. 4c), suggesting that the decreased transcriptional functioning of EIN3 was caused by the decreased EIN3 protein level. We also checked the levels of EIN3 mRNA, and no obvious change was observed in Col-0 seedlings upon treatment with CLE42p after 6 h (Fig. S11), suggesting that CLE42p regulates EIN3 function at the protein level. However, no change was observed in the protein levels of EIN3 in the transgenic plants 35S:EIN3-GFP/ein3 eill ebf1 ebf2 upon treatment with CLE42p (Fig. 4d). EIN3-binding F-box 1 (EBF1) and EBF2 are key components of the ethylene signaling pathway, with distinct but overlapping roles in mediating the degradation of EIN3 protein through the Ub/proteasome pathway (Guo \& Ecker, 2003; Potuschak et al., 2003; Gagne et al., 2004). These results revealed that EBF1/2 are required for the CLE42p-induced degradation of EIN3 protein. Next, we examined the levels of EIN3-binding F-box 1 (EBF1) protein after CLE42p treatment using 35S:EBF1-GFP/Col-0 plants with anti-GFP antibody. Western blot assays showed that the protein levels of EBF1 remarkably increased upon CLE42p treatment (Fig. 4e), suggesting that CLE42p promotes the degradation of EIN3 protein by inducing the accumulation of EBF proteins.

To further investigate the effect of CLE42 on EIN3 function, we performed qPCR analysis to examine the expression of ETHYLENE RESPONSE FACTOR1 (ERF1), a well-known downstream target of EIN3 and a positive regulator of ethylene signaling (Solano et al., 1998). Treatment with ACC increased the transcription of ERF1, which was significantly decreased by CLE42p (Fig. 4f). Interestingly, the expression levels of ERF1, ERF59/ORA59 and PDF1.2 were significantly downregulated in CLE42ox plants compared with Col-0 plants (Fig. S12), suggesting that endogenous CLE42 suppresses the activity of EIN3. We next performed chromatin immunoprecipitation (ChIP) analysis to determine the effect of CLE42 on the binding activity of EIN3 to ERF1 using 35S:EIN3GFP/Col-0 plants. As expected, EIN3 could directly bind to the promoter of ERF1, which was enhanced by ACC treatment (Fig. 4g). In contrast, CLE42p treatment evidently compromised the binding activity of EIN3 to ERF1 (Fig. 4g), suggesting that CLE42p suppresses the protein stability of EIN3 and in turn affects its activity. Together, these results suggest that CLE42 suppresses the stabilization and function of EIN3 by promoting the protein accumulation of EIN3-targeting F-box proteins. 
The above data pushed us to investigate how CEL42 affects the protein stability of EBF1. Interestingly, we found that the transcript level of $A C S 2$ was evidently suppressed in the leaves of CLE42ox compared with Col-0 (Fig. S13a). Moreover, ethylene production was evidently decreased in CLE42ox plants (Fig. S13b). In supporting these findings, exogenous application of CLE42p also suppressed the expressions of $A C S 2$ and the production of ethylene compared with the untreated plants (Fig. S13c,d). These results suggest that CLE42 promotes EBF1 protein accumulation possibly by suppressing endogenous ethylene production. Future studies are needed to investigate how CLE42 affects the expression of $A C S \mathrm{~s}$.

(a)

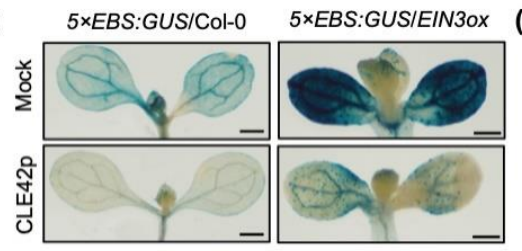

(b)
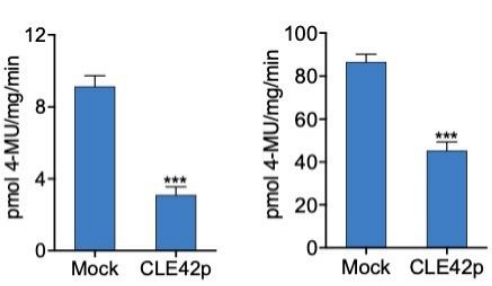

(c)

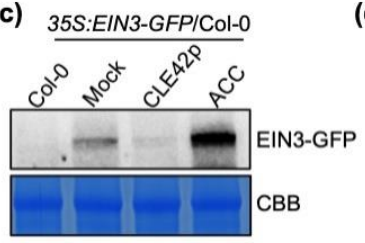

(d)

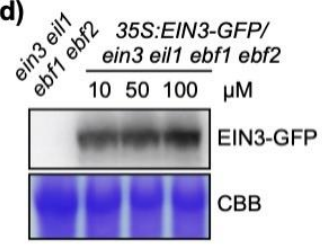

(e) 35S:EBF1-GFP/Col-0

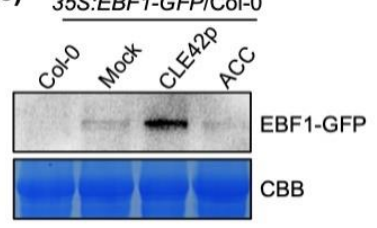

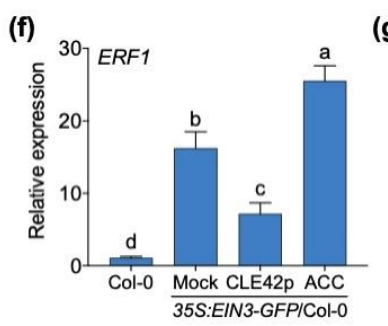

(g)

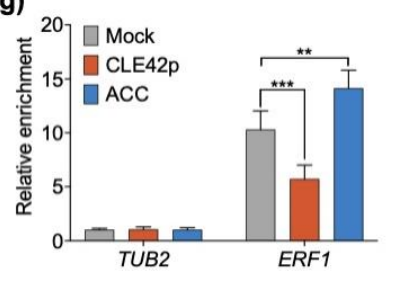

Fig. 4 CLE42 represses the function of EIN3. (a) Histochemical analysis of $5 \times E B S: G U S /$ Col-0 and $5 \times E B S: G U S / E I N 3 o x$ transgenic plants upon CLE42p treatment. Bars, $500 \mu \mathrm{m}$. (b) GUS activity in seedlings shown in (A) was calculated as picomoles of 4-MU per mg protein per min. *** $\mathrm{P}<0.001$, one-way ANOVA. (c) CLE42p reduces EIN3 protein accumulation in the wild type. Five-day-old seedlings were treated with $10 \mu \mathrm{M}$ CLE42p or ACC for $24 \mathrm{~h}$. Protein was extracted and subjected to immunoblots using anti-GFP antibody. Coomassie brilliant blue (CBB) staining was used as the protein loading control. (d) CLE42p-induced EIN3 protein degradation depends on EBF1/2. 35S:EIN3-GFP/ein3 eil1 ebf1 ebf2 seedlings treated with 10, 50 and $100 \mu \mathrm{M}$ CLE42p for $24 \mathrm{~h}$ were used for protein extraction. (e) CLE42p promotes EBF1 protein accumulation. Five-day-old seedlings were treated with $10 \mu \mathrm{M}$ CLE42p or ACC for $24 \mathrm{~h}$. Protein was extracted and subjected to immunoblots using anti-GFP antibody. Coomassie brilliant blue (CBB) staining was used as the protein loading control. (f) The transcript level of ERF1 in Col-0 and 35S:EIN3-GFP (EIN3ox) upon $10 \mu \mathrm{M}$ CLE42 or ACC treatment. The bars represent the standard deviation of three technical replicates. Different letters above the bars indicate statistically significant differences (adjusted $\mathrm{P}<$ 0.05, one-way ANOVA). (g) ChIP-qPCR was performed to examine the relative EIN3 binding to the ERF1 promoter under CLE42p treatment. An anti-GFP monoclonal antibody was used for DNA immunoprecipitation from 5-d-old EIN3ox transgenic plants. The relative enrichment of EIN3 binding to the ERF1 promoter was normalized to TUBULIN2 (TUB2). The bars represent the standard 
deviation of three technical replicates. Each experiment was repeated at least three times with similar results. ${ }^{* *} \mathrm{P}<0.01, * * * \mathrm{P}<0.001$, one-way ANOVA.

Loss-of-function of EIN3/EIL1 or overexpression of EBF1 suppresses the earlier senescence phenotypes of the cle42 mutant

To further explore the genetic regulatory relationship between CLE42 and EIN3/EIL1 in leaf senescence, we generated cle42 ein 3 eill plants by crossing the cle 42 plant with the ein 3 eill mutant, which was confirmed by the presence of triple response phenotypes and PCR sequencing (Table S1). Under long-day conditions, compared to Col-0, cle42 ein3 eill plants showed an obvious delayed senescence phenotype similar to ein 3 eill mutants, indicating that the accelerated leaf senescence phenotype of cle 42 mutants was effectively suppressed by the mutation of EIN3/EIL1 (Fig. 5a), which was confirmed by age-dependent changes in the chlorophyll content and Fv/Fm (Fig. 5b,c). Next, a dark-induced leaf senescence assay was performed using Col-0, cle42, ein3 eil1, and cle42 ein3 eil1 plants. Remarkably, ein3 eil1 strongly suppressed the earlier senescence phenotype of cle42 under darkness (Fig. 5d). The leaves of ein3 eill and cle42 ein3 eill showed higher levels of chlorophyll content and $\mathrm{Fv} / \mathrm{Fm}$ than Col-0 and cle42 leaves (Fig. 5e,f). These results suggest that mutation of EIN3 and EIL1 suppresses the earlier senescence phenotypes of cle42 plants.

Given that CLE42p promotes the protein accumulation of EBF1 and thus compromises EIN3 protein stability, we next analyzed whether overexpression of EBF1 could suppress the premature senescence phenotype of cle42 mutant. To this end, we examined the leaf senescence phenotypes of Col-0, cle42, cle42 EBF1ox, and EBF1ox plants. Compared with Col-0 and cle42 mutant, EBF1ox plants showed delayed senescence phenotypes with higher chlorophyll content and Fv/Fm, as well as lower levels of SAG12 gene expression (Fig. 5g-j), indicating that EBF1 delays leaf senescence. More interestingly, overexpression of EBF1 also suppressed the early senescence phenotypes of cle 42 mutant (Fig. 5g-j), suggesting that EBF1 acts downstream of CLE42 in the regulation of leaf senescence. Because CLE42 promotes EBF1 protein accumulation by suppressing ethylene biosynthesis, we examined whether loss of function of ACSs could rescue the early senescence phenotypes of cle42. Toward this end, the ACS octuple mutant (acs2-1 acs4-1 acs5-2 acs6-1 acs7-1 acs9-1 amiRacs8 acs11) (Tsuchisaka et al., 2009), eliminating the functional redundancy among multiple ACS genes, was used to generate octuple cle42 mutant using the CRISPR-Cas9 technology. As expectedly, mutation of multiple ACSs evidently suppressed the earlier senescence phenotypes of cle42 mutant (Fig. S14). Together, our results reveal that CLE42 delays leaf senescence through controlling the ACS-EBF-EIN3 cascade. 
(a)

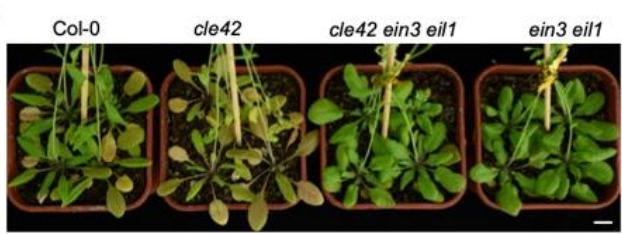

(d) $\quad \mathrm{Col}-0$

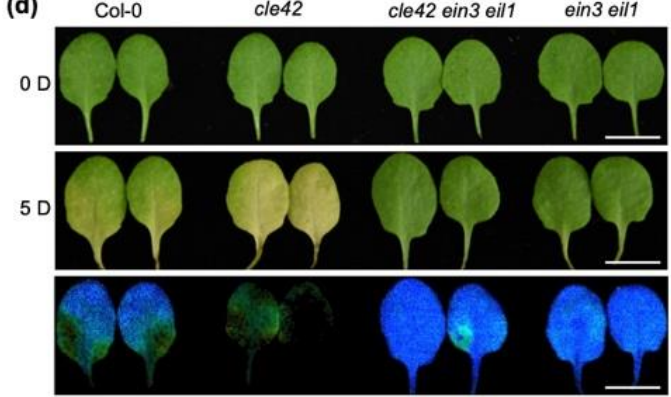

(g)

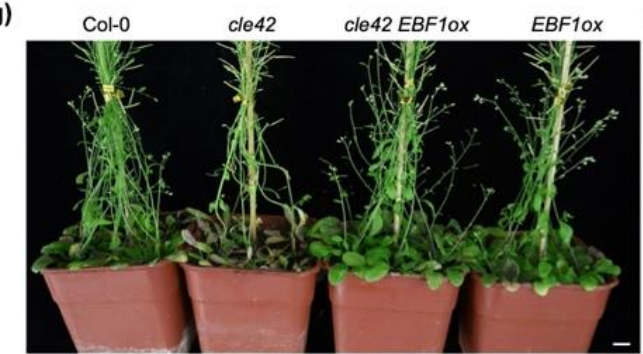

(b)

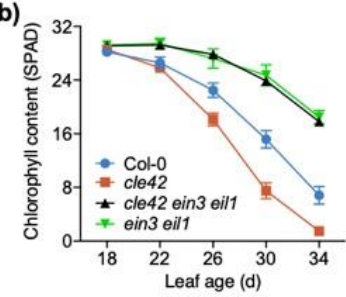

(e)

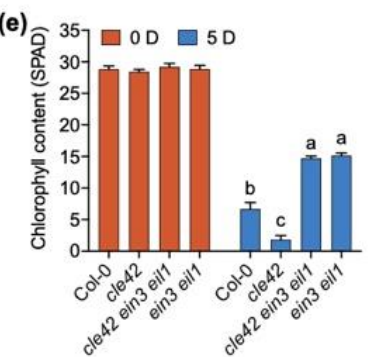

(i) (c)

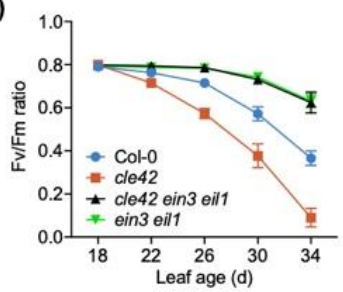

(f)

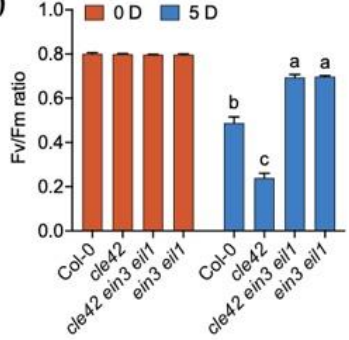

(j)
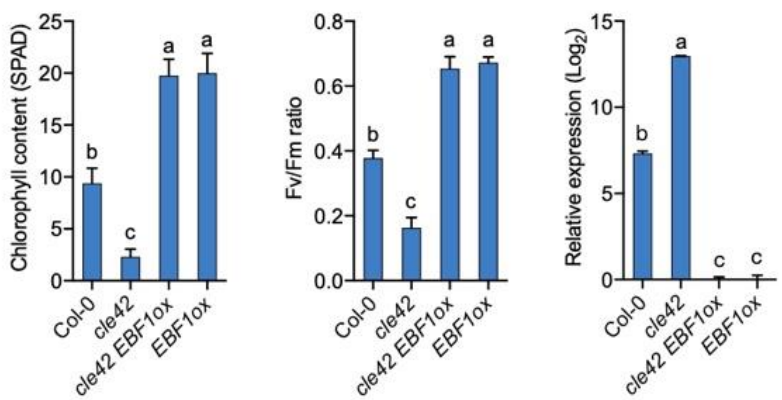

Fig. 5 Mutation of EIN3/EIL1 or overexpression of EBF1 suppresses the earlier senescence phenotypes of the cle42 mutant. (a) The senescence phenotypes of 40-d-old wild-type Col-0, cle42, ein3 eill, and cle42 ein 3 eill. Bar, $1 \mathrm{~cm}$. (b) and (c) chlorophyll content (b) and Fv/Fm ratio (c) in the leaves shown in (a) at the indicated age. The bars indicate the mean \pm SD $(n=20)$.

(d) The senescence phenotypes and Fv/Fm ratio of Col-0, cle42, ein3 eill, and cle42 ein3 eill leaves upon dark treatment for 4 days. (e) and (f) chlorophyll content (e) and Fv/Fm ratio (f) in the leaves shown in (d). (g) The senescence phenotypes of 45-d-old wild-type Col-0, cle42, cle42 EBF1ox and EBF1ox. Bar, $1 \mathrm{~cm}$. (h-j) Chlorophyll content (h), Fv/Fm ratio (i), and $S A G 12$ expression (j) in the fourth leaves shown in the plants in $(\mathrm{g})$. The bars indicate the mean $\pm \mathrm{SD}(\mathrm{n}=20$ for chlorophyll content and $\mathrm{Fv} / \mathrm{Fm}, \mathrm{n}=3$ for $\mathrm{qPCR}$ ). Different letters above the bars indicate statistically significant differences (adjusted $\mathrm{P}<0.05$, one-way ANOVA). 


\section{Discussion}

Leaf senescence is characterized by the transition from nutrient assimilation to remobilization and has a critical impact on agriculture (Gepstein, 2004; Avila-Ospina et al., 2014). As the last stage of leaf development, leaf senescence is not a passive and deteriorative process but a highly regulated and active process (Gepstein, 2004; Lim et al., 2007). The dramatic shift from a photosynthetically active organ to a senescent leaf is induced by a series of endogenous factors, such as age and plant hormones, as well as environmental cues, such as biotic and abiotic stresses (Gepstein, 2004; Guo \& Gan, 2005; Lim et al., 2007; Zhang et al., 2021a). Here, we found that CLE42, a peptide hormone of the CLE family in Arabidopsis (Cock \& McCormick, 2001), functions as a negative regulator of leaf senescence through integration with ethylene signaling (Fig. 1 and Fig. 4; Fig. S7), an endogenous modulator of plant aging (Abeles et al., 1988).

Peptide hormones participate in cell-to-cell communication to regulate plant growth and development in response to internal and external cues (Matsubayashi \& Sakagami, 2006). As the largest group of such peptides, CLE plays crucial roles in regulating meristem activity in shoots and roots as well as in vascular tissues (Cock \& McCormick, 2001; Fiers et al., 2005; Ito et al., 2006; Kondo et al., 2006). Recently, CLE14 was found to delay age-dependent and stress-induced leaf senescence by enhancing JUB1-mediated scavenge of reactive oxygen species (ROS) in Arabidopsis (Zhang et al., 2021b). Here, we found that mutation of CLE42 led to premature senescence, whereas overexpression of CLE42 resulted in prolonged plant longevity (Fig. 1). Exogenous application of chemically synthesized 12-aa peptides derived from CLE42 motifs functionally mimicked the overexpression of CLE42 and delayed age-dependent and dark-induced leaf senescence (Fig. 3; Fig. S6), supporting previous findings that peptides containing only the C-terminal CLE domain are sufficient to result in CLE activities (Fiers et al., 2005; Ito et al., 2006; Kondo et al., 2006). Expectedly, the earlier senescence phenotypes of cle42 mutant were also restored by an application of CLE41/44p under dark conditions (Fig. S6), indicative of the existence of function redundancy between CLE42 and CLE41/CLE44 in regulating leaf senescence. Although CLE41 and CLE44 are involved in the regulation of leaf senescence, their contribution seems relatively minor than that of CLE42 (Fig. 2b,c). One of the interpretations would be that the spatiotemporal expression patterns of CLE42 and CLE41/CLE44 are different (Fig. S2) (Yaginuma et al., 2011), which in turn cause different regulatory effects. We cannot exclude the possibility that the delay in leaf senescence mediated by CLE42 and CLE41/CLE44 is through different signaling pathways or possibly controlled by different receptors because they differ by one amino acid in the CLE motif. In supporting this possibility, previous studies find that CLE42 displays a weaker interaction with PXY compared to CLE41/CLE44 (Zhang et al., 2016), and CLE42 possessed partial activity of TDIF (Hirakawa et al., 2008). CLE42 differs from CLE41/CLE44 only in the second position but displayed 
a different effect on leaf senescence, raising the possibility that the non-conserved residues may allow CLE peptides to be distinguished by their receptors and regulates different developmental process.

Secreted CLE peptides stimulate intracellular signaling through plasma membrane-localized receptors. The binding of a CLE peptide to its receptor triggers various downstream signaling events. CLE42 and CLE41/CLE44 have been shown to bind the LRR-RLK receptor TDR/PXY (Fisher \& Turner, 2007; Hirakawa et al., 2008). Similar to the cle41 cle42 cle44 triple mutant, mutation in $T D R / P X Y$ also resulted in reduced plant longevity (Fig.2), suggesting that the CLE41/CLE42/CLE44PXY signaling module functions as a negative regulator of plant aging. Arabidopsis genome contains two PXY-like genes, PXL1 and PXL2 (Fisher \& Turner, 2007), which function redundantly in regulating vascular tissue development (Etchells et al., 2013). However, no additional contribution of PXL1/PXL2 in leaf senescence was observed when comparing pxy and pxy pxl1 pxl2 (Fig. S5), implicating that PXY is involved in regulating leaf ageing. Compared with pxy mutant, the single mutant cle 41 , cle 42 or cle 44 displayed relatively weaker senescence phenotype (Fig. 2); however, the cle41 cle42 cle44 mutant exhibited stronger senescence phenotype than pxy (Fig. 2b-d; Fig. S5), suggesting that the role of CLE41/CLE42/CLE44 in the regulation of leaf senescence might be partially PXY-independent manner. In supporting this hypothesis, overexpression of CLE42 could slightly rescue the senescence phenotypes of pxy (Fig. 3d-f). Previous studies found that CLE9/10 regulate two different processes by being perceived by two distinct receptor systems. CLE9/10 participates in regulating stomatal development through associating with receptor kinase HAESALIKE 1 (HSL1), whereas CLE9/10 regulates xylem development through interacting with BARELY NO MERISTEM (BAMs) (Qian et al., 2018). Therefore, more research is needed in the future to explore whether CLE41/42/44 interact with other receptors to control leaf senescence.

CLE peptides regulate a number of biological processes by integrating phytohormone signaling in plants (Wang et al., 2015). CLE10 inhibits protoxylem formation by suppressing the expression of ARR5 and ARR6, two negative regulators of cytokinin signaling (Kondo et al., 2011). Overexpression of CLE14 or CLE2O reduces cytokinin content and impacts root growth, which can be rescued by the exogenous application of cytokinin (Meng \& Feldman, 2010). Here, we found that CLE42 is involved in multiple factors-induced senescence, including age, darkness, plant hormones (JA and SA) and $\mathrm{H}_{2} \mathrm{O}_{2}$ (Fig.1 and 3; Fig. S3). Interestingly, CLE14 also delays these factors-induced leaf senescence by governing $\mathrm{H}_{2} \mathrm{O}_{2}$ homeostasis (Zhang et al., 2021b). These findings suggest that CLE14 and CLE42 regulate leaf senescence probably through similar pathways. Future studies such as the generation of cle14 cle42 double mutants need to be conducted to test this possibility. The CLE25 peptide modulates ABA biosynthesis by inducing NCED3 expression in the leaves to regulate stomatal closure under drought conditions (Takahashi et al., 2018). CLE26 affects the primary root protophloem by modulating auxin signaling (Czyzewicz et al., 2015). CLE40 represses cytokinin 
505 signaling by suppressing gene expression in signaling and biosynthesis (Pallakies \& Simon, 2014). 506 In this study, we found that several hormone signaling pathways, including JA, SA and ethylene, 507 were suppressed in CLE42-overexpressing plants through transcriptome analysis (Fig.S7), suggesting 508 that CLE42 delays leaf senescence by suppressing positive modulators of plant aging. Application of 509 the CLE42 peptide reduced the protein accumulation of EIN3 by stabilizing EBF1 and suppressed 510 the function of EIN3 (Fig. 4). Interestingly, expression levels of ethylene biosynthesis gene ACS2 511 were suppressed by treatment with CLE42p, resulting in a decrease in ethylene production (Fig. S13). 512 Correspondingly, overexpression of CLE42 evidently suppressed the response to low concentrations 513 of ACC under dark conditions. In contrast, mutation of CLE42 enhanced the response to ethylene 514 (Fig. S10). Moreover, loss of function of EIN3/EIL1, overexpression of EBF1, or mutation of $A C S \mathrm{~s}$ 515 suppressed the earlier senescence of the cle42 mutant (Fig. 5; Fig. S14), suggesting that CLE42 delays 516 leaf senescence by controlling the ACS-EBF-EIN3 cascade. Additionally, overexpression of CLE42 517 attenuated the triple response as well as delayed leaf senescence in the presence of ethylene. 518 Conversely, cle42 mutants showed enhanced responses and early senescence (Fig. S3, S10, and S11). 519 Taken together, our results suggested that CLE42-PXY signaling module controls leaf senescence by 520 communicating with ethylene pathway. It was also noticed that CLE41/42/44, especially CLE42, 521 plays a prominent role in response to low concentrations of ethylene (Fig. S10, S11). Given that plant produces a low level of ethylene during normal and stress conditions, which is sufficient for plant growth, development, and survival (Catala et al., 2014; Johnson and Ecker, 1998; Vong et al., 2019;

524 Yoon and Chen, 2017), our data suggested that CLE42 might be an important player in plant growth 525 and development through interacting with ethylene pathway. 


\section{Acknowledgments}

528 We gratefully thank Dr. Peter Etchells (Durham University, UK) for the pxy and pxy pxl1 pxl2 529 mutants. This work was supported by the National Natural Science Foundation of China (32170345 530 and 31970196 to ZL), and the National Key Research and Development Program of China (No. 531 2019YFA0903904 to H.G.).

\section{Author contributions}

533 ZL conceived the project and designed the experiments; HG and XX designed some of the 534 experiments; ZY carried out most of the experiments; ST, YG, and CK prepared DNA construct, 535 measured chlorophyll content and Fv/Fm; TI and SS developed CLE CRISPR/Cas9 mutant 536 collection; HLW performed bioinformatics analysis; QY performed WB analysis; ZL and YZ wrote 537 the manuscript with input from all co-authors. All authors agreed on the final version of the 538 manuscript.

539 Data availability

540 RNA-Seq data are available in the Sequence Read Archive (SRA, https://www.ncbi.nlm.nih.gov/sra/) 541 with the accession number PRJNA804857. 
bioRxiv preprint doi: https://doi.org/10.1101/2022.02.27.481379; this version posted March 1,2022 . The copyright holder for this preprint (which was not certified by peer review) is the author/funder. All rights reserved. No reuse allowed without permission.

\section{References}

Abeles FB, Dunn LJ, Morgens P, Callahan A, Dinterman RE, Schmidt J. 1988. Induction of 33$\mathrm{kD}$ and $60-\mathrm{kD}$ peroxidases during ethylene-induced senescence of cucumber cotyledons. Plant Physiol 87(3): 609-615.

Alonso JM, Stepanova AN, Solano R, Wisman E, Ferrari S, Ausubel FM, Ecker JR. 2003. Five components of the ethylene-response pathway identified in a screen for weak ethylene-insensitive mutants in Arabidopsis. Proc Natl Acad Sci U S A 100(5): 2992-2997.

Avila-Ospina L, Moison M, Yoshimoto K, Masclaux-Daubresse C. 2014. Autophagy, plant senescence, and nutrient recycling. J Exp Bot 65(14): 3799-3811.

Betsuyaku S, Sawa S, Yamada M. 2011. The function of the CLE peptides in plant development and plant-microbe interactions. Arabidopsis Book 9: e0149.

Bleecker AB, Estelle MA, Somerville C, Kende H. 1988. Insensitivity to ethylene conferred by a dominant mutation in Arabidopsis thaliana. Science 241(4869): 1086-1089.

Clough SJ, Bent AF. 1998. Floral dip: a simplified method for Agrobacterium-mediated transformation of Arabidopsis thaliana. Plant J 16(6): 735-743.

Cock JM, McCormick S. 2001. A large family of genes that share homology with CLAVATA3. Plant Physiol 126(3): 939-942.

Cutler SR, Ehrhardt DW, Griffitts JS, Somerville CR. 2000. Random GFP::cDNA fusions enable visualization of subcellular structures in cells of Arabidopsis at a high frequency. Proc Natl Acad Sci U S A 97(7): 3718-3723.

Czyzewicz N, Shi CL, Vu LD, Van De Cotte B, Hodgman C, Butenko MA, De Smet I. 2015. Modulation of Arabidopsis and monocot root architecture by CLAVATA3/EMBRYO SURROUNDING REGION 26 peptide. J Exp Bot 66(17): 5229-5243.

Etchells JP, Provost CM, Mishra L, Turner SR. 2013. WOX4 and WOX14 act downstream of the PXY receptor kinase to regulate plant vascular proliferation independently of any role in vascular organisation. Development 140(10): 2224-2234.

Etchells JP, Provost CM, Turner SR. 2012. Plant vascular cell division is maintained by an interaction between PXY and ethylene signalling. PLoS Genet 8(11): e1002997.

Etchells JP, Smit ME, Gaudinier A, Williams CJ, Brady SM. 2016. A brief history of the TDIFPXY signalling module: balancing meristem identity and differentiation during vascular development. New Phytol 209(2): 474-484.

Fiers M, Golemiec E, Xu J, van der Geest L, Heidstra R, Stiekema W, Liu CM. 2005. The 14amino acid CLV3, CLE19, and CLE40 peptides trigger consumption of the root meristem in Arabidopsis through a CLAVATA2-dependent pathway. Plant Cell 17(9): 2542-2553.

Fiers M, Ku KL, Liu CM. 2007. CLE peptide ligands and their roles in establishing meristems. Curr Opin Plant Biol 10(1): 39-43.

Fisher K, Turner S. 2007. PXY, a receptor-like kinase essential for maintaining polarity during plant vascular-tissue development. Curr Biol 17(12): 1061-1066.

Fletcher JC. 2020. Recent advances in Arabidopsis CLE peptide signaling. Trends Plant Sci 25(10): 1005-1016.

Gagne JM, Smalle J, Gingerich DJ, Walker JM, Yoo SD, Yanagisawa S, Vierstra RD. 2004. Arabidopsis EIN3-binding F-box 1 and 2 form ubiquitin-protein ligases that repress ethylene action and promote growth by directing EIN3 degradation. Proc Natl Acad Sci U S A 101(17): 6803-6808.

Gan S, Amasino RM. 1995. Inhibition of leaf senescence by autoregulated production of cytokinin. Science 270(5244): 1986-1988.

Gao X, Guo Y. 2012. CLE peptides in plants: proteolytic processing, structure-activity relationship, and ligand-receptor interaction. J Integr Plant Biol 54(10): 738-745.

Gepstein S. 2004. Leaf senescence--not just a 'wear and tear' phenomenon. Genome Biol 5(3): 212.

Guo H, Ecker JR. 2003. Plant responses to ethylene gas are mediated by SCF(EBF1/EBF2)dependent proteolysis of EIN3 transcription factor. Cell 115(6): 667-677. 
bioRxiv preprint doi: https://doi.org/10.1101/2022.02.27.481379; this version posted March 1,2022 . The copyright holder for this preprint (which was not certified by peer review) is the author/funder. All rights reserved. No reuse allowed without permission.

Guo Y, Gan S. 2005. Leaf senescence: signals, execution, and regulation. Curr Top Dev Biol 71: 83112.

Hirakawa Y, Sawa S. 2019. Diverse function of plant peptide hormones in local signaling and development. Curr Opin Plant Biol 51: 81-87.

Hirakawa Y, Shinohara H, Kondo Y, Inoue A, Nakanomyo I, Ogawa M, Sawa S, Ohashi-Ito K, Matsubayashi Y, Fukuda H. 2008. Non-cell-autonomous control of vascular stem cell fate by a CLE peptide/receptor system. Proc Natl Acad Sci U S A 105(39): 15208-15213.

Hohmann-Marriott MF, Blankenship RE. 2011. Evolution of photosynthesis. Annu Rev Plant Biol 62: $515-548$.

Hortensteiner S, Feller U. 2002. Nitrogen metabolism and remobilization during senescence. $J$ Exp Bot 53(370): 927-937.

Ito Y, Nakanomyo I, Motose H, Iwamoto K, Sawa S, Dohmae N, Fukuda H. 2006. Dodeca-CLE peptides as suppressors of plant stem cell differentiation. Science 313(5788): 842-845.

Jefferson RA, Kavanagh TA, Bevan MW. 1987. GUS fusions: beta-glucuronidase as a sensitive and versatile gene fusion marker in higher plants. EMBO J 6(13): 3901-3907.

Kim HJ, Hong SH, Kim YW, Lee IH, Jun JH, Phee BK, Rupak T, Jeong H, Lee Y, Hong BS, et al. 2014. Gene regulatory cascade of senescence-associated NAC transcription factors activated by ETHYLENE-INSENSITIVE2-mediated leaf senescence signalling in Arabidopsis. J Exp Bot 65(14): 4023-4036.

Kim J, Woo HR, Nam HG. 2016. Toward systems understanding of leaf senescence: An integrated multi-omics perspective on leaf senescence research. Mol Plant 9(6): 813-825.

Kim JH, Woo HR, Kim J, Lim PO, Lee IC, Choi SH, Hwang D, Nam HG. 2009. Trifurcate feedforward regulation of age-dependent cell death involving miR164 in Arabidopsis. Science 323(5917): 1053-1057.

Kondo T, Sawa S, Kinoshita A, Mizuno S, Kakimoto T, Fukuda H, Sakagami Y. 2006. A plant peptide encoded by CLV3 identified by in situ MALDI-TOF MS analysis. Science 313(5788): 845-848.

Kondo Y, Hirakawa Y, Kieber JJ, Fukuda H. 2011. CLE peptides can negatively regulate protoxylem vessel formation via cytokinin signaling. Plant Cell Physiol 52(1): 37-48.

Li Z, Peng J, Wen X, Guo H. 2013. Ethylene-insensitive3 is a senescence-associated gene that accelerates age-dependent leaf senescence by directly repressing miR164 transcription in Arabidopsis. Plant Cell 25(9): 3311-3328.

Li Z, Zhang Y, Zou D, Zhao Y, Wang HL, Zhang Y, Xia X, Luo J, Guo H, Zhang Z. 2020. LSD 3.0: a comprehensive resource for the leaf senescence research community. Nucleic Acids Res 48(D1): D1069-D1075.

Li Z, Zhao Y, Liu X, Peng J, Guo H, Luo J. 2014. LSD 2.0: an update of the leaf senescence database. Nucleic Acids Res 42(Database issue): D1200-1205.

Lim PO, Kim HJ, Nam HG. 2007. Leaf senescence. Annu Rev Plant Biol 58: 115-136.

Lim PO, Nam HG. 2005. The molecular and genetic control of leaf senescence and longevity in Arabidopsis. Curr Top Dev Biol 67: 49-83.

Matsubayashi Y, Sakagami Y. 2006. Peptide hormones in plants. Annu Rev Plant Biol 57: 649-674.

Meng L, Feldman LJ. 2010. CLE14/CLE20 peptides may interact with CLAVATA2/CORYNE receptor-like kinases to irreversibly inhibit cell division in the root meristem of Arabidopsis. Planta 232(5): 1061-1074.

Mou S, Zhang X, Han Z, Wang J, Gong X, Chai J. 2017. CLE42 binding induces PXL2 interaction with SERK2. Protein Cell 8(8): 612-617.

Nam HG. 1997. The molecular genetic analysis of leaf senescence. Curr Opin Biotechnol 8(2): 200207.

Oh SA, Park JH, Lee GI, Paek KH, Park SK, Nam HG. 1997. Identification of three genetic loci controlling leaf senescence in Arabidopsis thaliana. Plant J 12(3): 527-535.

Pallakies H, Simon R. 2014. The CLE40 and CRN/CLV2 signaling pathways antagonistically control root meristem growth in Arabidopsis. Mol Plant 7(11): 1619-1636. 
bioRxiv preprint doi: https://doi.org/10.1101/2022.02.27.481379; this version posted March 1, 2022. The copyright holder for this preprint (which was not certified by peer review) is the author/funder. All rights reserved. No reuse allowed without permission.

Pearce G, Strydom D, Johnson S, Ryan CA. 1991. A polypeptide from tomato leaves induces wound-inducible proteinase inhibitor proteins. Science 253(5022): 895-897.

Potuschak T, Lechner E, Parmentier Y, Yanagisawa S, Grava S, Koncz C, Genschik P. 2003. EIN3-dependent regulation of plant ethylene hormone signaling by two Arabidopsis $\mathrm{F}$ box proteins: EBF1 and EBF2. Cell 115(6): 679-689.

Qiu K, Li Z, Yang Z, Chen J, Wu S, Zhu X, Gao S, Gao J, Ren G, Kuai B, et al. 2015. EIN3 and ORE1 accelerate degreening during ethylene-mediated leaf senescence by directly activating chlorophyll catabolic genes in Arabidopsis. PLoS Genet 11(7): e1005399.

Saleh A, Alvarez-Venegas R, Avramova Z. 2008. An efficient chromatin immunoprecipitation (ChIP) protocol for studying histone modifications in Arabidopsis plants. Nat Protoc 3(6): 10181025 .

Schmittgen TD, Livak KJ. 2008. Analyzing real-time PCR data by the comparative C(T) method. Nat Protoc 3(6): 1101-1108.

Smit ME, McGregor SR, Sun H, Gough C, Bagman AM, Soyars CL, Kroon JT, Gaudinier A, Williams CJ, Yang X, et al. 2020. A PXY-mediated transcriptional network integrates signaling mechanisms to control vascular development in Arabidopsis. Plant Cell 32(2): 319-335.

Solano R, Stepanova A, Chao Q, Ecker JR. 1998. Nuclear events in ethylene signaling: a transcriptional cascade mediated by ETHYLENE-INSENSITIVE3 and ETHYLENERESPONSE-FACTOR1. Genes Dev 12(23): 3703-3714.

Stepanova AN, Yun J, Likhacheva AV, Alonso JM. 2007. Multilevel interactions between ethylene and auxin in Arabidopsis roots. Plant Cell 19(7): 2169-2185.

Takahashi F, Suzuki T, Osakabe Y, Betsuyaku S, Kondo Y, Dohmae N, Fukuda H, YamaguchiShinozaki K, Shinozaki K. 2018. A small peptide modulates stomatal control via abscisic acid in long-distance signalling. Nature 556(7700): 235-238.

Tsuchisaka A, Yu G, Jin H, Alonso JM, Ecker JR, Zhang X, Gao S, and Theologis A. 2009. A combinatorial interplay among the 1-aminocyclopropane-1-carboxylate isoforms regulates ethylene biosynthesis in Arabidopsis thaliana. Genetics 183(3): 979-1003.

Uauy C, Distelfeld A, Fahima T, Blechl A, Dubcovsky J. 2006. A NAC Gene regulating senescence improves grain protein, zinc, and iron content in wheat. Science 314(5803): 1298-1301.

Ueda H, Kusaba M. 2015. Strigolactone regulates leaf senescence in concert with ethylene in arabidopsis. Plant Physiol 169(1): 138-147.

Wang C, Dai S, Zhang ZL, Lao W, Wang R, Meng X, Zhou X. 2021. Ethylene and salicylic acid synergistically accelerate leaf senescence in Arabidopsis. J Integr Plant Biol 63(5): 828-833.

Wang G, Zhang G, Wu M. 2015. CLE peptide signaling and crosstalk with phytohormones and environmental stimuli. Front Plant Sci 6: 1211.

Woo HR, Chung KM, Park JH, Oh SA, Ahn T, Hong SH, Jang SK, Nam HG. 2001. ORE9, an F-box protein that regulates leaf senescence in Arabidopsis. Plant Cell 13(8): 1779-1790.

Woo HR, Kim HJ, Lim PO, Nam HG. 2019. Leaf Senescence: Systems and dynamics aspects. Annu Rev Plant Biol 70: 347-376.

Woo HR, Kim HJ, Nam HG, Lim PO. 2013. Plant leaf senescence and death - regulation by multiple layers of control and implications for aging in general. J Cell Sci 126(Pt 21): 4823-4833.

Woo HR, Kim JH, Nam HG, Lim PO. 2004. The delayed leaf senescence mutants of Arabidopsis, ore1, ore3, and ore 9 are tolerant to oxidative stress. Plant Cell Physiol 45(7): 923-932.

Woo HR, Koo HJ, Kim J, Jeong H, Yang JO, Lee IH, Jun JH, Choi SH, Park SJ, Kang B, et al. 2016. Programming of plant leaf senescence with temporal and inter-organellar coordination of transcriptome in Arabidopsis. Plant Physiol 171(1): 452-467.

Yaginuma H, Hirakawa Y, Kondo Y, Ohashi-Ito K, Fukuda H. 2011. A novel function of TDIFrelated peptides: promotion of axillary bud formation. Plant Cell Physiol 52(8): 1354-1364.

Yamaguchi YL, Ishida T, Sawa S. 2016. CLE peptides and their signaling pathways in plant development. J Exp Bot 67(16): 4813-4826.

Yamaguchi YL, Ishida T, Yoshimura M, Imamura Y, Shimaoka C, Sawa S. 2017. A Collection of mutants for CLE-peptide-encoding genes in Arabidopsis generated by CRISPR/Cas9-mediated 
bioRxiv preprint doi: https://doi.org/10.1101/2022.02.27.481379; this version posted March 1, 2022. The copyright holder for this preprint (which was not certified by peer review) is the author/funder. All rights reserved. No reuse allowed without permission.

gene targeting. Plant Cell Physiol 58(11): 1848-1856.

Yu X, Xu Y, Yan S. 2021. Salicylic acid and ethylene coordinately promote leaf senescence. J Integr Plant Biol 63(5): 823-827.

Zhang H, Lin X, Han Z, Qu LJ, Chai J. 2016. Crystal structure of PXY-TDIF complex reveals a conserved recognition mechanism among CLE peptide-receptor pairs. Cell Res 26(5): 543-555.

Zhang Y, Gao Y, Wang HL, Kan C, Li Z, Yang X, Yin W, Xia X, Nam HG, Li Z, et al. 2021a. Verticillium dahliae secretory effector PevD1 induces leaf senescence by promoting ORE1mediated ethylene biosynthesis. Mol Plant. https://doi.org/10.1016/j.molp.2021.07.014.

Zhang Z, Liu C, Li K, Li X, Xu M, Guo Y. 2021b. CLE14 functions as a "brake signal" suppressing age-dependent and stress-induced leaf senescence through promoting JUB1-mediated ROS scavenge in Arabidopsis. Mol Plant https://doi.org/10.1016/j.molp.2021.09.006.

Zuo J, Niu QW, Chua NH. 2000. Technical advance: An estrogen receptor-based transactivator XVE mediates highly inducible gene expression in transgenic plants. Plant J 24(2): 265-273. 


\section{Supporting Information}

714 Fig. S1 qPCR analysis of CLE42 expression in the fourth leaves of 3-week-old Col-0 and CLE42ox 715 plants.

716 Fig. S2 The expression of CLE41/42/44 decreases during leaf aging.

717 Fig. S3 CLE42 negatively modulates senescence-regulating signal-triggered leaf senescence.

718 Fig. S4 Genotyping analysis of cle41 cle42 cle44 by sequencing.

719 Fig. S5 The cle41 cle42 cle44 shows earlier senescence phenotypes than the receptor mutants.

720 Fig. S6 CLE41/44p restored the early senescence phenotype of the cle42 mutant under dark 721 conditions.

722 Fig. S7 Gene ontology analysis of differentially regulated genes in CLE42ox plants.

723 Fig. S8 The expression of senescence-associated genes in Col-0 and CLE42ox plants.

724 Fig. S9 Overexpression of CLE42 caused ethylene insensitivity under low-concentration ACC 725 conditions.

726 Fig. S10 Loss of function of CLE42 results in the most prominent triple responses compared with 727 CLE41/CLE44.

728 Fig. S11 The expression of EIN3 in Col-0 upon CLE42p treatment.

729 Fig. S12 The expression of downstream target genes of ethylene signaling in Col-0 and CLE42ox 730 plants.

731 Fig. S13 CLE42 suppresses ethylene biosynthesis.

732 Fig. S14 The ACS octuple mutant suppressed the earlier senescence phenotypes of cle42 mutant. 733 Table S1. Primers used in the study.

734 Dataset S1. Differentially expressed genes in CLE42ox compared to Col-0. 